\title{
Individualized dosing of aminoglycosides in neonates: mission accomplished or work in progress?
}

\author{
John N. van den Anker • Karel Allegaert
}

Received: 31 May 2009/Accepted: 23 June 2009 / Published online: 7 July 2009

(C) The Author(s) 2009. This article is published with open access at Springerlink.com

\section{Dear Editor,}

We have read with great interest the paper of Sherwin et al. on individualized dosing of amikacin based on a population pharmacokinetic and -dynamic (PKPD) study in 80 neonates [1]. To the very best of our knowledge, this is the first PD study (outcome indicator sepsis) of aminoglycosides in neonates. We fully support the clinical need to evaluate both PK and PD of drugs, including aminoglycosides in neonates. The recent review on aminoglycosides in neonates in this journal hereby illustrates that clinical pharmacologists are aware of and interested in the specific needs and characteristics of this patient population [2]. We are, however, intrigued by the dosing suggestions formulated by the authors: $15 \mathrm{mg} / \mathrm{kg}$ at 36-h intervals for neonates with a postmenstrual age (PMA) $\leq 28$ weeks, $14 \mathrm{mg} / \mathrm{kg}$ at 24-h intervals for neonates

\footnotetext{
J. N. van den Anker $(\bowtie)$

Division of Pediatric Clinical Pharmacology,

Children's National Medical Center,

111 Michigan Avenue, NW,

Washington, DC, USA

e-mail: JVandena@cnmc.org

J. N. van den Anker

Departments of Pediatrics, Pharmacology and Physiology,

George Washington University School of Medicine

and Health Sciences,

Washington, DC, USA

J. N. van den Anker

Department of Pediatrics,

Erasmus MC- Sophia Children's Hospital,

Rotterdam, The Netherlands

K. Allegaert

Neonatal Intensive Care Unit, University Hospitals Leuven, Leuven, Belgium
}

with a PMA between 29 and 36 weeks, and $15 \mathrm{mg} / \mathrm{kg}$ at 24-h intervals for neonates with a $P M A \geq 37$ weeks [1].

Firstly, if we apply the clearance model proposed $\left[\right.$ clearance $\left.=0.23 \times(\text { current weight } / 2)^{0.691} \times(\mathrm{PMA} / 40)^{3.23}\right]$ to the range of neonates included (weight $0.44-4.4 \mathrm{~kg}$; PMA 24-41 weeks), clearance ranges from 0.0155 to $0.428 \mathrm{~L} / \mathrm{h}$ ( 30 -fold interindividual variability). This extensive interindividual variability in clearance is not reflected in the dosing recommendations formulated. As aminoglycoside clearance reflects glomerular filtration rate (GFR), it is to be anticipated that maturational clearance displays a continuum, instead of the dichotomous pattern: $50 \%$ reduction in amikacin administration $\leq 28$ weeks, and only a very limited additional interindividual variability $(<10 \%)$ between 29 weeks and full-term neonates seems not to be in line with developmental maturation of renal clearance capacity. Although it is likely that the current dosing suggestions fit best with the available data set, it is a likely that the current suggestions do not yet fully reflect the interindividual variability in clearance of aminoglycosides in neonates [2-4].

Secondly, based on the fact that interindividual variability in any aminoglycoside clearance reflects interindividual variability in GFR, we urgently call for prospective validation of the current and other clearance models suggested. As clearance of aminoglycoside reflects the interindividual variability in GFR, cross-validation studies should include not only different cohorts but also different aminoglycosides. We hereby would like refer to the netilmicin study in neonates recently published by the same group, where the clearance model seems to be based on the same covariates model $\left[\right.$ netilmicin clearance $=0.192 \times(\text { current weight } / 2)^{1.35} \times$ (PMA/40 $)^{1.03}$ ] but with different exponential factors [5].

Besides collaboration between different centers and groups, such cross-validation studies necessitate robust data sets that include other relevant covariates in addition to 
PMA or weight alone. We miss information on the existence of a patent ductus arteriosus (PDA), on the exposure to indomethacin or ibuprofen to induce closure of the PDA, or on the presence of growth restriction. as it has been clearly demonstrated that these covariates in part explain the interindividual variability in aminoglycoside clearance in neonates [6-8]. Similarly, we would like to mention the relevance of the technique used to determine creatinine level in the blood. For decades we have known that the Jaffe method introduces additional errors when used in neonates because of interferences by, e.g., ketoacids, bilirubin, or cephalosporins. It really makes a difference to use an enzymatic method in this age group [9].

In conclusion, we would like to encourage the authors and other research groups interested to critically re-evaluate their single cohort aminoglycoside clearance model and recommendations. In addition to single population studies, cross-validation studies, including extrapolation of a given clearance model to data sets with other aminoglycosides, are urgently needed to unveil the underlying maturational GFR and its covariates. This will necessitate collaborative efforts and can only be based on robust data sets including covariates of potential relevance and should not be limited to weight or PMA alone.

Acknowledgements The clinical research of J.N. van den Anker is supported by grants of the National Institute of Health (NICCHD 1U10HD45993, NCRR 1K24RR019729). The clinical research of K. Allegaert is supported by the Fund for Scientific Research, Flanders (Belgium) (FWO Vlaanderen) by a Fundamental Clinical Investigatorship $(1800209 \mathrm{~N})$ and an research grant $(1506409 \mathrm{~N})$.
Open Access This article is distributed under the terms of the Creative Commons Attribution Noncommercial License which permits any noncommercial use, distribution, and reproduction in any medium, provided the original author(s) and source are credited.

\section{References}

1. Sherwin CM, Svahn S, van der Linden A, Broadbent RS, Medlicott NJ, Reith DM (2009) Individualised dosing of amikacin in neonates: a pharmacokinetic/pharmacodynamic analysis. Eur J Clin Pharmacol. doi:10.1007/s00228-009-0637-4

2. Pacifici GM (2009) Clinical pharmacokinetics of aminoglycosides in the neonate: a review. Eur J Clin Pharmacol 65:419-427

3. Allegaert K, Anderson BJ (2006) Interindividual variability of aminoglycoside pharmacokinetics in preterm neonates at birth. Eur J Clin Pharmacol 62:1011-1012

4. De Hoog M, Mouton JW, van den Anker JN (2005) New dosing strategies for antibacterial agents in the neonate. Semin Fetal Neonatal Med 10:185-194

5. Sherwin CM, Broadbent RS, Medlicott NJ, Reith DM (2008) Individualising netilmicin dosing in neonates. Eur J Clin Pharmacol 64:1201-1208

6. Van den Anker JN, Kearns GL (1997) Gentamicin pharmacokinetics in neonates with patent ductus arteriosus. Crit Care Med 25:1933-1934

7. Allegaert K, Anderson BJ, van den Anker JN, Vanhaesebrouck S, de Zegher F (2007) Renal drug clearance in preterm neonates: relation to prenatal growth. Ther Drug Monit 29:284-291

8. Allegaert K, Cossey V, Langhendries JP, Naulaers G, Vanhole C, Devlieger H, van Overmeire B (2004) Effects of co-administration of ibuprofen-lysine on the pharmacokinetics of amikacin in preterm infants during the first days of life. Biol Neonate 86:207-211

9. Van den Anker JN (1997) Renal function in preterm infants. Eur J Pediatr 156:583-584 